QIONGFANG ZENG, Ph.D. ${ }^{1}$

E-mail: qfzeng@csu.edu.cn YINGGUI ZHANG, Ph.D. ${ }^{1}$

(Corresponding author)

E-mail:ygzhang@csu.edu.cn

DINGYOU LEI, Ph.D. ${ }^{1}$

(Corresponding author)

E-mail: ding@csu.edu.cn

${ }^{1}$ School of Traffic and Transportation Engineering

Central South University, Changsha 410075, China
Science in Traffic and Transport

Preliminary Communication

Submitted: 24 Sep. 2016

Accepted: 7 Apr. 2017

\title{
AN EXPERIMENTAL ANALYSIS ON DISPATCHING RULES FOR THE TRAIN PLATFORMING PROBLEM IN BUSY COMPLEX PASSENGER STATIONS
}

\begin{abstract}
This paper presents the scheduling models for train platforming problem (TPP) by using mixed integer linear programming and job shop scheduling theory. First, the operation procedures and scheduled time adjustment costs of different train types specific to busy complex passenger stations are explicitly represented. Second, a multi-criteria scheduling model (MCS) for TPP without earliness and tardiness time window (ETTW) and a time window scheduling model (TWS) with ETTW for TPP are proposed. Third, various dispatching rules were designed by incorporating the dispatcher experiences with modern scheduling theory and a rule-based metaheuristic to solve the above model is presented. With solution improvement strategies analogous to those used in practice by dispatchers, the realistic size problems in acceptable time can be solved.
\end{abstract}

\section{KEY WORDS}

busy complex passenger station; train platforming problem; job shop scheduling theory; dispatching rule;

\section{INTRODUCTION}

In recent years, rapid development of high-speed railway network and its integration with the existing passenger railway network has enhanced the intricacy of train platforming problem in busy complex passenger stations which has sustained high density in arrivals and departures, complexity in station layout and variety in train types. Automation level for the train routing assignment process in busy complex passenger stations is still low. In the past decades, train platforming problem (TPP) has been researched significantly to increase the work efficiency of dispatchers who manage dense traffic in busy complex passenger stations. Given the station layout and the arrival and departure times in draft timetable, TPP refers to the creation of platform track assignment plan in a busy complex station by finding a conflict-free resource assignment plan. According to Sels et al. (2014), the train routing problem can be categorized into strategic, tactical and operational/real-time level [1]. Many researchers such as Dewilde et al. (2014) and Zhou et al. (2016) consider a combination and iteration of strategic and tactical level, namely, the feedback of unassigned trains on a tactical level to the following adjustment or even cancellation of their desired arrival or departure times at strategic level [2, 3]. However, Cacchiani et al. (2014) pointed out that an integrated approach is generally hopeless for real-world instances due to the complexity of train timetabling problem (TTP) and the TPP [4].

Most of the existing literature that dealt with train scheduling by using job shop scheduling theory (JSST) are focused on the timetabling and routing of railway corridors and networks, where the nodes between the block sections (intersections, meet points, stations, etc.) are implicitly or simply modelled as some special kind of block sections (e.g. station section). Mascis et al. (2002), D'Ariano et al. (2007) and Samà et al. (2015) applied the alternative graph (AG) as an effective tool to model train routing problem as blocking and no-wait job shop scheduling problems [5-7]. Quite different from blocking time theory in the AG model, most complex stations run with the route-locking sectional-release interlocking system, and unscheduled stops are generally not allowed along consecutive track sections of the interlocking area (Corman et al. 2009). According to Corman et al. (2009), the disaggregated model in which track segment is regarded as a no-store machine is optimistic approximation of sectional-release route locking operations; the aggregated model that groups together the track sections is pessimistic approximation of sectional-release route locking operations [8]. In this paper, the TPP problem in busy complex stations as a job-shop problem with additional constrains that explain exactly the 
sectional-release route-locking operations in complex stations are identified. Also, different operations of different train types conducted in complex passenger stations and different scheduled time adjustment penalty cost function of different train types are represented, which is necessary for track-circuited formulation of TPP specific to complex stations.

Some researchers including Carey et al. (2003) and Carey et al. (2007) developed heuristics analogous to those successfully adopted by station dispatchers using manual methods [9, 10]. Station dispatchers usually assign one train to one track at a time, and then a conflict check is conducted to avoid conflict between the train and all the previously assigned trains. Although Carey et al. (2003) and Carey et al. (2007) experimented with different train orders such as random order, chronological order and reverse chronological order when choosing the next train to consider for scheduling in complex passenger stations, other orders are ruled out. In the station with relatively low density of train arrivals and departures and simple station layout, researchers such as Carey et al. (2003) think that the train order (dispatching rule) when choosing the next train to consider for scheduling is not important, especially when heuristic methods are used to further optimize the solution based on a certain dispatching rule. In this paper, we call the solution based on certain dispatching rules as rule solution (RS) in abbreviation. In complex passenger stations with a large number of platform tracks, most inbound and outbound routes are partially coincident and have a lot of intersections. Nevertheless, the number of unassigned trains increases with the growing density of arrivals and departures in complex passenger stations because the platform track and route resources are more limited. Thus, the order in which trains are considered when assigning platform tracks tend to affect not only the next few trains and may cause higher degree of delay propagation in these complex and busy station area. As for complex and busy stations, the RS may be infeasible in practice due to the high density of train arrivals and departures and complex station layout, and the RS is often used as initial solution of heuristic algorithm which aims to further improve the feasibility and optimize the solution. The near optimal solution of heuristic algorithm is generally independent of its initial solution without time limit. As the reassignment of platform tracks to trains are time-critical in real-time level, metaheuristic approaches are only permitted to run in limited iterations in order to meet the time requirement (Fang, 2015) [11]. Here, we will formulate the TPP problem in busy complex stations into special cases of job-shop problem based on fine granularity, and we will also exploit the structure of the job shop scheduling problem to get the best possible and feasible platform track assignment plan within limited computation time. In particular, various dispatching rules are designed by incorporating the dispatcher experiences with modern scheduling theory and proposed metaheuristics to improve the solution quality. Further, we compare the impact of dispatching rules on good quality solutions of metaheuristics in acceptable computation times.

The remainder of this paper is organized as follows. Section 2 defines the related notions and depicts characteristics of technical operations in busy complex passenger stations. Section 3 identifies train TPP as the job shop problem and sets up an MCS and related constrains for the TPP. Section 4 extends the MCS to real-time level by presenting a TWS for TPP with ETTW. Section 5 designs the dispatching rules and introduces a rule-based metaheuristic algorithm to solve the above model. In Section 6, numerical experiments and the comparisons of the results are given. The paper concludes with a summary and discussion of some extensions for further research in Section 7.

\section{CONCEPTS, ASSUMPTIONS AND NOTATIONS}

In recent years, a lot of researchers such as Lusby et al. (2013) and Pellegrini et al. (2014) proposed resource-based (i.e. track-circuits) formulations and heuristics [12, 13]. However, none of the existing papers have a clear representation of different operating procedures of various train types specific to complex passenger stations. This section gives a detailed description of the concepts, assumptions and the operation processes of different train types in busy complex railway passenger stations.

\subsection{Concept and assumption illustration in our TPP version}

Passenger railway stations use a route-locking sectional-release interlocking system to ensure safety of train movements. When a train is at a point near a station, all track-circuits in the inbound route and platform track assigned to the train are claimed or reserved at the same moment in advance. Similarly, all the track-circuits of the outbound route assigned to a certain train are claimed at an instant before the train departs from the station. Most importantly, the track-circuits within the interlocking area are released one by one once the train rear has exited. Here, we define the occupation time interval of a resource as the time interval that starts from the claim time instant to the release time instant of the resource. The dispatchers usually proactively coordinate the speed of trains on open track and do not let trains stop or wait at the interlocking area, and the claim and release time instants of resources can be derived by subtracting or adding the setup and running time of the 
chosen routes or track-circuits from the actual arrival and departure time.

According to different train services and technical operational processes, passenger trains can be classified into originating passenger trains (ORPTs), terminating passenger trains (TEPTs), turnaround passenger trains (TUPTs), and through passenger trains (THPTs). Specifically, THPTs can be classified into non-stop through passenger trains (NSTHPTs) and stop through passenger trains (STHPTs) according to whether or not they will stop at the station.

Here, we have the following assumptions:

1) Multiple alternative inbound (outbound) routes lead the train from the entry (exit) point to a platform track.

2) The platform track assignment of trains is not fixed, and we can choose one platform track out of several alternative platform tracks.

Table 1 - Notations for general subscripts and input parameters

\begin{tabular}{|c|c|}
\hline Symbol & Description \\
\hline$x_{i}$ & Arrival time of train $i$ in original timetable \\
\hline$x_{i}^{*}$ & Arrival time of train $i$ in actual timetable \\
\hline$y_{i}$ & Departure time of train $i$ in original timetable \\
\hline$y_{i}^{*}$ & Departure time of train $i$ in actual timetable \\
\hline$\tau_{\mathrm{sf}}$ & Standard service time of originating passenger trains \\
\hline$\tau_{z d}$ & Standard service time of terminating passenger trains \\
\hline$J_{i} \in \mathbf{J}$ & $\begin{array}{l}\text { Set of passenger trains indexed by } i . n \text { is the total number of trains within a given period } \\
\text { of the timetable. }\end{array}$ \\
\hline $\mathrm{J}_{\text {or }}$ & Set of originating passenger trains \\
\hline $\mathbf{J}_{t e}$ & Set of terminating passenger trains \\
\hline $\mathbf{J}_{t u}$ & Set of turnaround passenger trains \\
\hline $\mathbf{J}_{n s}$ & Set of non-stop through passenger trains \\
\hline $\mathbf{J}_{\text {st }}$ & Set of stop through passenger trains \\
\hline$M_{k} \in \mathbf{M}$ & Set of all platform tracks indexed by $k . K_{m}$ is the total number of platform tracks. \\
\hline$Z_{p} \in \mathbf{P}$ & Set of all platforms indexed by $p . K_{p}$ is the total number of platforms. \\
\hline$R_{l} \in \mathbf{R}$ & Set of all routes indexed by $I . K_{r}$ is the total number of routes. \\
\hline $\mathrm{S}_{q} \in \mathrm{Set}$ & Set of all track-circuits indexed by $q . K_{q}$ is the total number of track-circuits. \\
\hline$\omega_{i}$ & Weight of train $i$. \\
\hline$C_{i p}$ & Time cost of inconvenience of train $i$ if train $i$ is allocated to the not-preferred platform $p$. \\
\hline$J-M$ & Mapping relationships between trains and platform tracks. \\
\hline M - P & Mapping relationships between platform tracks and platforms. \\
\hline$M-R$ & Mapping relationships between platform tracks and routes \\
\hline R - S - H & Mapping relationships between routes and track-circuits. \\
\hline $\mathbf{J}_{t u}^{a}-\mathbf{J}_{t u}^{d}$ & Mapping relationships between ATUPTs and DTUPTs. \\
\hline$\theta_{i}$ & Limitation: number of the alternative platform tracks that are allowed to be claimed by train $i$ \\
\hline$Q_{i}$ & Set of alternative platform tracks of train $i$ \\
\hline$Q_{i k}^{a}$ & Set of alternative inbound routes for train $i$ ending at platform track $k$ \\
\hline$Q_{i k}^{d}$ & Set of alternative outbound routes for train $i$ starting from platform track $k$ \\
\hline$K_{l}$ & Number of track-circuits that make up route I \\
\hline Set $_{1}$ & $\begin{array}{l}\text { Set of track-circuits claimed sequentially by a train when the train goes through route } I \text {. } \\
\text { Set } S e t_{l}=\left\{S_{1}^{\prime}, S_{2}^{\prime}, \ldots S_{h}^{\prime}, \ldots, S_{K_{1}}^{\prime}\right\} \text { is sequenced according to the interlocking chart. }\end{array}$ \\
\hline$L_{h}^{\prime}$ & 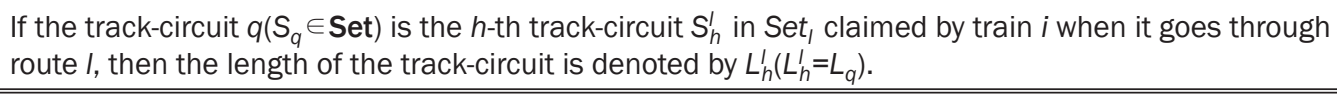 \\
\hline
\end{tabular}


Table 2 - Notation for data created during processing

\begin{tabular}{|c|l||}
\hline Symbol & \multicolumn{1}{|c||}{ Description } \\
\hline \hline$J^{\prime}$ & Processing sequencing list, $J^{\prime}=\left\{J_{1}^{\prime}, \ldots, J_{j}^{\prime}, J_{j}^{\prime} ; \ldots, J_{n}^{\prime}\right\}$. For any different trains $J_{i}^{\prime}$ and $J_{j}^{\prime}(i \neq j)$, is prior to $J_{i}^{\prime}$ \\
\hline$t_{z}^{k}$ & The $z$-th occupation time interval of platform track $k$ \\
\hline$\Gamma$ & Unassigned train set \\
\hline$S(i, k)$ & Variable for storing the claim (Start) time instant of platform track $k\left(k \in Q_{i k}\right)$ by the train $i$. \\
\hline$F(i, k)$ & Variable for storing the release (Finish) time instant of platform track $k\left(k \in Q_{i k}\right)$ by the train $i$. \\
\hline$S\left(i, I, h, o_{i k l}\right)$ & Variable for storing the claim time instant of track-circuit $h\left(h \in \operatorname{Set}_{1}\right)$ in route $I\left(I \in Q_{i k}^{\text {a }} \cup Q_{i k}^{d}\right)$ by train $i$. \\
\hline$F\left(i, I, h, o_{i k}\right)$ & Variable for storing the release time instant of track-circuit $h\left(h \in \operatorname{Set}_{1}\right)$ in route $I\left(I \in Q_{i k}^{\text {a }} \cup Q_{i k}^{d}\right)$ by train $i$. \\
\hline$\rho_{k}$ & Total occupation times of platform track $k$ \\
\hline
\end{tabular}

Table 3 - Decision variables

\begin{tabular}{||c|l||}
\hline \hline Symbol & \multicolumn{1}{|c||}{ Description } \\
\hline \hline$o_{i k}$ & 0-1 binary variable. If platform track $k$ is claimed by train $i$, then $o_{i k}=2 ;$ otherwise $o_{i k}=0$. \\
\hline$o_{i k l}$ & $\begin{array}{l}\text { Route variable. If route } I \text { is claimed by train as an inbound route ending at platform track } k \text {, then } \\
o_{i k l}=1 ; \text { if route is claimed by train } i \text { as an outbound route starting from platform track } k \text {, then } o_{i k l}=2 ; \\
\text { otherwise } o_{i k l}=0 .\end{array}$ \\
\hline$o_{i k l q}$ & $\begin{array}{l}0-1 \text { binary track-circuit variable. If track-circuit } q \text { is claimed by train } i \text { as part of route } I \text { that is connect- } \\
\text { ed with platform track } k, \text { then } o_{i k l q}=1 ; \text { otherwise } o_{i k l q}=0 .\end{array}$ \\
\hline$o_{i p}$ & $0-1$ binary platform variable. If platform $p$ is occupied by train $i$, then $o_{i p}=1 ;$ otherwise $o_{i p}=0$. \\
\hline$D_{i}$ & $\begin{array}{l}0-1 \text { binary variable of delayed train. If train } J_{i} \text { is not assigned to a complete route through the station, } \\
\text { then } D_{i}=1 ; \text { otherwise } D_{i}=0 .\end{array}$ \\
\hline
\end{tabular}

3) We assume that each platform track can accommodate one train at any time.

4) Carriage formation of passenger trains is relatively fixed. The mapping relationships between ATUPTs and DTUPTs can be acquired from the carriage turnover plan. An arrival turnaround passenger train (ATUPT) terminating at the station changes into a corresponding departure turnaround passenger train (DTUPT) that will go back to the originating station by maintaining carriages in the assigned platform track and changing the locomotive position. To better formulate the problem and calculate the platform track occupation time interval, we will integrate ATUPT and DTUPT that share the same carriages and the same platform track into one TUPT. The TUPT's arrival time is the arrival time of its corresponding ATUPT in timetable, and the TUPT's departure time is the departure time of its corresponding DTUPT in the timetable.

5) After a TEPT stopping at its assigned platform track, all the passengers in this train will alight from the train within a standard service time, and shunting locomotives should be arranged to place the TEPT in the passenger carriages servicing depot or railway passenger technical station. The TEPTs may be broken up and coupled in the technical station to make up ORPTs. Then, shunting locomotives should be arranged to take ORPTs out of the technical station to their assigned platform track. After boarding all the passengers within the standard service time, the ORPT will start up and depart from the station. In this paper, the interferences of placing-in operations for TEPTs, taking-out operations for ORPTs and locomotive-changing operation for ATUPTs are not considered because the arrival and departure operations are given higher priority.

\subsection{Notations}

This section presents the following notations used in the problem formulation. Tables 1 and 2 list general subscripts and input parameters. Table 3 describes decision variables.

\section{MCS WITHOUT TIME-WINDOW CONSTRAINT}

The job-shop scheduling problem is the problem of allocating machines to competing jobs over time and defining starting times and completing times of all the operations. If we regard the processing of trains (jobs) at the platform tracks (machines) as operations, then all trains follow the same operations through the interlocking area. One train can only be assigned with one platform track while one platform track can only process one train at the same time, which corresponds to the assumption when we consider jobs on parallel machines. Track circuits and platforms can only be occupied by one train at occupation time interval and can be assigned to new trains once they are released. 
Thus, the station resources such as track-circuits and platforms can be regarded as constrained renewable resources. In the end, the TPP without ETTW can be regarded as job-shop scheduling problem with parallel machines and renewable resources constrains.

A job $i$ is characterized by its operation processing time $p_{i}$. If the transversal of trains on platform tracks corresponds to the operations in job shop problem, the processing time of train $i$ can be denoted by $p_{i}=y_{i}^{*}-x_{i}^{*}$ Each job has attributes such as ready time $r_{i}$ and due date $c_{i}$. The ready time for train $J_{i}$ can be formulated as follows:

$r_{i}= \begin{cases}y_{i}^{*}-\tau_{s f} & \text { for ORPT } \\ x_{i}^{*} & \text { for others }\end{cases}$

The due date for train $i$ can be formulated as follows:

$c_{i}= \begin{cases}x_{i}^{*}+\tau_{z d} & \text { for TEPT } \\ x_{i}^{*} & \text { for NSTHPT } \\ y_{i}^{*} & \text { for others }\end{cases}$

Therefore, the multi-criteria scheduling mode (MCS) for TPP is denoted by the 3-field problem classification $\alpha|\beta| \gamma$ (Graham et al., 1979) as shown in Equation 3.

(MCS)

$$
\begin{aligned}
P m= & \mid J_{i}, r_{i}, p_{i}, c_{i}, \omega_{i}, \vartheta_{i}, J-M, J_{t u}^{a}-J_{t u}^{d}, \\
& M-P, M-R, R-S-H \mid F\left(f_{1}, f_{2}, f_{3}\right)
\end{aligned}
$$

\subsection{Objective function}

The efficiency of a production system is measured by the total flow time of all jobs in the system (Alagöz et al., 2003) [14]. A number of cost criteria such as the total lateness and the number of jobs in the system are closely related to the total flow time of a schedule. In this paper, total tardiness has been chosen as the most important cost criteria, and the first sub-objective is minimizing the weighted number of delayed trains as shown in Equation 4.

$f_{1}=\sum_{i=1}^{n} \omega_{1} D_{1}$

As explained in Caprara et al. (2011), the total time cost of inconvenience to passengers if trains are allocated to not-preferred platforms can be shown in Equation 5 [15].

$f_{2}=\sum_{i=1}^{n} \sum_{p=1}^{K p} c_{i p} 0_{i p}$

However, the above preference may cause overuse of preferred platforms and platform tracks and idling losses of some not-preferred platforms and platform tracks. From the station dispatchers' perspective, the balanced use of resources such as platforms and track-circuits can reduce the delay possibility due to the maintenance time window for wearing switches and platform facilities and save idling losses of some not-preferred platforms and platform tracks. Thus, specific to the large number of platform tracks in complex stations, the objective of balanced use of resources is presented. If platform track $k$ is claimed by train $i$ and it is the $z$-th occupation time of platform track $k$ by trains, then the z-th occupation time interval of platform track $k$ is expressed by Equation 6. The balanced use level of station resources in railway passenger station is expressed by Equation 7.

$t_{z}^{k}=F(i, k)-S(i, k)$

$f_{3}=\sum_{k=1}^{K_{m}}\left(\sum_{z=1}^{\rho_{k}} t_{z}^{k}-\frac{1}{K_{m}} \sum_{k=1}^{K_{m}} \sum_{z=1}^{\rho_{k}} t_{z}^{k}\right)^{2}$

Loss of time due to delay of jobs are denoted by $\chi$.These three objectives are further combined by weighted summation into one hierarchical multi-objective Function 8 where $\lambda_{1}, \lambda_{2}$ and $\lambda_{3}$ give the importance of each sub-objective.

$F\left(f_{1}, f_{2}, f_{3}\right)=\lambda_{1} \chi f_{1}+\lambda_{2} f_{2}+\lambda_{3} f_{3}$

\subsection{Constraints}

The occupation time interval of train $i$ claiming track-circuit $q$ on route $/$ that connected to platform track $k$ and the occupation time interval of another train $j$ claiming the same track-circuit $q$ on route $e$ that connected to platform track $d$ should be non-overlapping, otherwise conflicts in track-circuit $q$ may occur. This constraint is expressed by Equation 9.

$$
\begin{aligned}
& {\left[S\left(i, l, q, o_{i k l}\right), F\left(i, l, q, o_{i k l}\right)\right] \cap} \\
& {\left[S\left(j, e, q, o_{j d e}\right), F\left(J_{j}, e, q, o_{j d e}\right)\right]=\phi} \\
& i \neq j, \forall o_{i k l q}=o_{j d e q}=1, \forall S_{q} \in \operatorname{Set}
\end{aligned}
$$

The occupation time interval of train $i$ claiming platform track $k$ and the occupation time interval of another train $j$ claiming the same platform track $k$ should be non-overlapping, otherwise conflicts in platform track $k$ may occur. This constraint is expressed by Equation 10.

$[S(i, k), F(i, k)] \cap[S(j, k), F(j, k)]=\phi$

$$
i \neq j, \forall O_{i k}=o_{j k}=1, \forall M_{k} \in \mathbf{M}
$$

Constraint that each train $i$ in set $\mathbf{J}$ can claim one platform track at the most is expressed by Equation 11 .

$$
\sum_{k=1}^{K_{m}} O_{i k}=1 \quad \forall J_{i} \in J
$$

Constraint that each train in set $\mathbf{J}$ can claim one platform at the most is expressed by Equation 12 . 


$$
\sum_{p=1}^{K_{p}} O_{i p}=1 \quad \forall J_{i} \in \mathbf{J}
$$

\section{TWS BASED ON TIME WINDOW CONSTRAINT}

By using scheduling algorithm based on dispatching rules, RS of MCS can be achieved directly in a short time. The dispatchers usually change the platform track and route assignment or swap train processing list orders to increase the RS feasibility and further improve the solution quality. In case that solution improvement strategies are not effective enough in improving RS feasibility, the dispatcher will co-ordinate the speed of trains on open track to retime the arrival or departure time of unassigned trains within ETTWs to increase the possibility of acquiring a feasible platform track assignment plan. Many other researchers such as Caimi et al. (2012) and Dewilde et al. (2013) proposed different ways to retime the actual timetable in passenger station and took minimizing the costs due to violating original timetable as an important objective $[16,17]$. Here, we will explicitly represent the retiming costs of different types of trains and make our objective more reasonable.

If the dwelling time of ORPTs or TEPTs on platform track exceeds the allowable time window which reserved redundant time for the shunting locomotives to place-in and take-out carriages, the station operation will be affected badly. Similar to the earliness time window of NORPTs and the tardiness time window of NTEPTs, the following two definitions are given.

Definition 3.1 Similar earliness time window is used to describe the allowable time window for the platform track in the reception-departure yard to receive the carriages of ORPTs that are taken out of the passenger technical station.

Definition 3.2 Similar tardiness time window is used to describe the allowable time window for the carriages of TEPTs to depart from the platform track in the reception-departure yard and go to the passenger technical station.

Let $E_{a i}$ and $T_{a i}$ denote the earliness and tardiness of the actual arrival time $x_{i}^{*}$, respectively, and let and denote the earliness and tardiness of the actual departure time $y_{i}^{*}$, respectively, as shown in Equations 13 and 14.

$$
\begin{aligned}
& \left|x_{i}^{*}-x_{i}\right|= \begin{cases}E_{a i} & x_{i}^{*} \leq x_{i} \\
T_{a i} & x_{i}^{*}>x_{i}\end{cases} \\
& \left|y_{i}^{*}-y_{i}\right|= \begin{cases}E_{d i} & y_{i}^{*} \leq y_{i} \\
T_{d i} & y_{i}^{*}>y_{i}\end{cases}
\end{aligned}
$$

Furthermore, let $\beta_{a i}$ denote the unit earliness and tardiness penalty cost (UPC) of actual arrival time of NORPTs, and $\beta_{d i}$ denote the UPC of actual departure time of NTEPTs. Also, let $\beta_{z i}$ denote the UPC for the time of TEPTs' carriages to depart from the reception-departure yard, and $\beta_{\mathrm{si}}$ denote the UPC for the time of ORPTs' carriages to arrive at the reception-departure yard.

Therefore, the total penalty cost $f_{\text {total }}$ caused by earliness or tardiness is expressed by Equations 15-19. The $\varepsilon$ is a binary variable of whether the train is running ahead of or behind the given timetable.

$$
\begin{aligned}
& f_{\text {total }}=f_{a i}+f_{d i}+f_{s i}+f_{z i} \\
& f_{a i}=\sum_{\forall J_{i} \in J_{t e} \cup J_{t u} \cup J_{s t}} \beta_{a i}\left(\varepsilon E_{a i}+(1-\varepsilon) T_{a i}\right) \\
& f_{d i}=\sum_{\forall J_{i} \in J_{o r} \cup J_{t u} \cup J_{s t}} \beta_{d i}\left(\varepsilon E_{d i}+(1-\varepsilon) T_{d i}\right) \\
& f_{s i}=\sum_{\forall J_{i} \in J_{o r}} \beta_{s i}\left(\varepsilon E_{a i}+(1-\varepsilon) T_{a i}\right) \\
& f_{z i}=\sum_{\forall J_{i} \in J_{t e}} \beta_{z i}\left(\varepsilon E_{d i}+(1-\varepsilon) T_{d i}\right)
\end{aligned}
$$

Cacchiani et al. (2014) pointed out that passenger connections and mapping relationship between pairs of trains shared carriage are implicitly described already by the departure and arrival times in original timetable [4]. If the connected trains are retimed within a certain ETTW, the UPC $\beta_{a i}, \beta_{d i}, \beta_{s i}$ and $\beta_{z i}$ are all proportional to the train weight $\omega_{i}$ and inversely proportional to its limitation $\theta_{i}$. Also, the increasing rates of UPC are growing gradually with the increasing of the earliness or tardiness of arrival or departure times. If train's actual arrival or departure time is beyond allowable given ETTW, then UPC tends to be infinite and unacceptable because passenger connections, carriage and crew management are severely affected. Based on the characteristics of technical operations for railway passenger transport business, the variation trends for UPC with the change of actual arrival or departure time $t$ for a given train are illustrated in Figure 1.

The illustration shows the different UPC variation trends for different train types when their arrival and departure times are adjusted within ETTWs.

In Figure $1 a$, for any NORPT $i,\left[a_{0 i}, b_{0 i}\right]$ are the ETTW of arrival time. In Figure $1 b$, for any NTEPT $i,\left[a_{1 i}, b_{1 i}\right]$ is ETTW of departure time. Generally, a train is not allowed to depart from station ahead of the timetable and is allowed to appropriately delay its departure time. Thus, the allowable earliness time interval $\left[a_{1 i}, y_{i}\right]$ is reduced to zero, and the ETTW is further rewritten as $\left[y_{i}, b_{1 i}\right]$.

For any ORPT $i,\left[a_{2 i}, b_{2 i}\right],\left[a_{2 i}^{\prime}, a_{2 i}\right]$ and $\left[b_{2 i}, b_{2 i}^{\prime}\right]$ are the punctuality time window, SETW and STTW of 


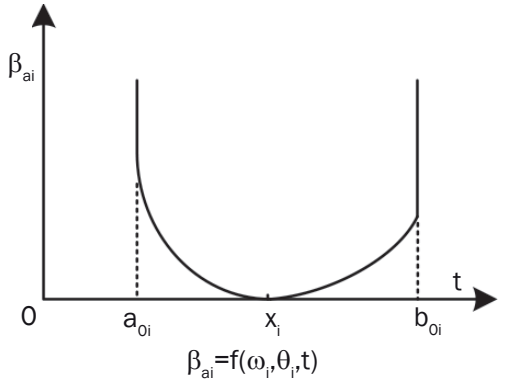

a) UPC vs. arrival time for NORPTs

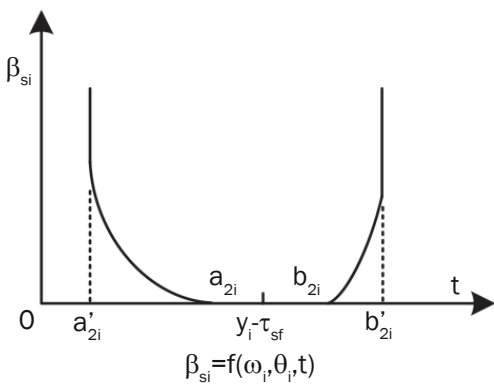

c) UPC vs. arrival time for ORPTs

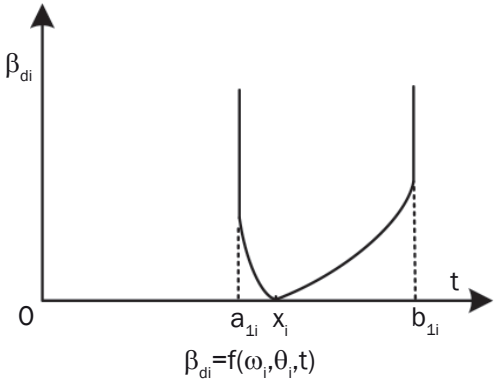

b) UPC vs. departure time for NTEPTs

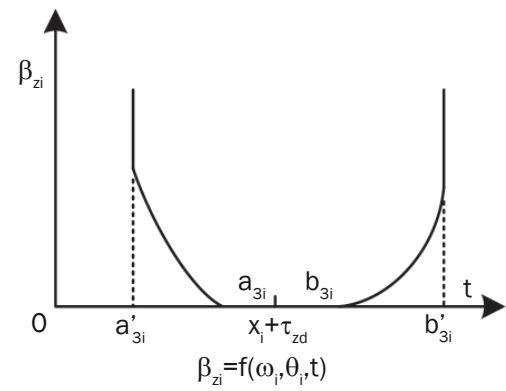

d) UPC vs. departure time for TEPTs

Figure 1 - UPC variation trends for different train types within time windows

arrival time for carriages of ORPT, respectively in Figure 1c. For any TEPT $i,\left[a_{3 i}, b_{3 i}\right],\left[a_{3 i}^{\prime}, a_{3 i}\right]$ and $\left[b_{3 i}, b_{3 i}^{\prime}\right]$ are the punctuality time window, SETW and STTW of departure time for carriages of TEPT, respectively in Figure 1d. As for ORPTs and TEPTs, the UPC are zero and the following operations of passenger carriages will be guaranteed if their carriages are placed in or taken out by locomotives within punctuality time window.

$$
\begin{aligned}
& P m \mid J_{i}, r_{i}, p_{i}, c_{i}, \omega_{i}, \vartheta_{i}, J-M, J_{t u}^{a}-J_{t u}^{d}, M-P, \\
& M-R, R-S-H, E T T W \mid \frac{F\left(f_{1}, f_{2}, f_{3}\right)}{\lambda_{4} f_{\text {total }}}
\end{aligned}
$$$$
f_{a i}=\left\{\begin{array}{r}
\sum_{\forall J_{i} \in J_{t e} \cup J_{t u} \cup J_{s t}}\left(\frac{\xi_{1} \omega_{i}}{\theta_{i}} E_{a i}^{2}\right) a_{0 i} \leq t<x_{i} \\
\sum_{\forall J_{i} \in \mathrm{J}_{\text {te }} \cup J_{t u} \cup_{s t}}\left(\frac{\xi_{2} \omega_{i}}{\theta_{i}} T_{a i}^{2}\right) x_{i} \leq t \leq b_{0 i} \\
t<a_{0 i}, t>b_{0 i}
\end{array}\right.
$$$$
f_{d i}=\left\{\begin{array}{rl}
t & t<y_{i}, t>b_{1 i} \\
\sum_{\forall J_{i} \in J_{o r} \cup J_{t u} \cup J_{s t}}\left(\frac{\xi_{3} \omega_{i}}{\theta_{i}} T_{d i}^{2}\right) y_{i} \leq t \leq b_{1 i}
\end{array}\right.
$$$$
f_{s i}=\left\{\begin{array}{cc}
\sum_{\forall J_{i} \in J_{o r}}\left(\frac{\xi_{4} \omega_{i}}{\theta_{i}} E_{a i}^{2}\right) & a_{2 i}^{\prime} \leq t<a_{2 i} \\
0 & a_{2 i} \leq t \leq b_{2 i} \\
\sum_{\forall J_{i} \in J_{o r}}\left(\frac{\xi_{5} \omega_{i}}{\theta_{i}} T_{a i}^{2}\right) & b_{2 i}<t \leq b_{2 i}^{\prime} \\
+\infty & t<a_{2 i}^{\prime}, t>b_{2 i}^{\prime}
\end{array}\right.
$$$$
f_{z i}=\left\{\begin{array}{lc}
\sum_{\forall J_{i} \in J_{t e}}\left(\frac{\xi_{6} \omega_{i}}{\theta_{i}} E_{d i}^{2}\right) & a_{3 i}^{\prime} \leq t<a_{3 i} \\
0 & a_{3 i} \leq t \leq b_{3 i} \\
\sum_{\forall J_{i} \in J_{t e}}\left(\frac{\xi_{7} \omega_{i}}{\theta_{i}} T_{d i}^{2}\right) & b_{3 i}<t \leq b_{3 i}^{\prime} \\
+\infty & t<a_{3 i}^{\prime}, t>b_{3 i}^{\prime}
\end{array}\right.
$$
parameters for UPC. shown in Equation 24.

(TWS)

\section{RULE-BASED METAHEURISTIC} limited computation time.

\subsection{Dispatching rules}

The TPP can be further regarded as just-in-time scheduling problem with ETTW on parallel machines with one of the objectives being to minimize the total weighted earliness and tardiness penalty cost subject to the constant renewable resource constrains as

This section gives some dispatching rules to solve the above MCS and TWS. A rule-based metaheuristics is also proposed in this section to improve the feasibility of RS and achieve the good quality solution within

For the basic scheduling problems, there are some basic dispatching rules for different objectives. However, some dispatching rules refined from dispatcher's experience also give us important inspiration to solve the TPP problem. In practical experience, the station dispatchers usually give priority to assignment of trains with the smallest number of alternative platform tracks, namely, the smallest limitation first dispatching rule (SL). Thus, those trains with bigger weight or 
smallest number of alternative platform tracks will be given higher priority to occupy limited platform tracks and route resources, which gives higher possibility of reducing the number of unassigned trains.

Combining the rules elicited from the dispatcher experience with the dispatching rules for classic scheduling problems, we propose various dispatching rules as follows: earliest due date first (EDD), first in first out (FIFO), shortest processing time first (SPT), weighted SPT first (WSPT), weighted discounted SPT first (WDSPT) (Pinedo, 2008) [18], biggest weight first (BW), smallest limitation first (SL), FIFO-EDD, EDD-FIFO, WSPT-EDD, WSPT-FIFO-EDD, SL-FIFO-EDD, BW-SL-FIFOEDD, SL-BW-FIFO-EDD and $\omega / \theta$-FIFO-EDD. (Symbol "-" means both rules are adopted at the same time and the former one is prior to the latter one.)

\subsection{Metaheuristics}

The complexity of the feasibility problem of train routing problem is studied in detail in Kroon et al. (1997) [19]. In particular, it turns out that the feasibility problem is solvable in polynomial time if each train has at the most two available route-deviation combinations, and it is NP-complete if each train can have three available route-deviation combinations. Introducing additional constraints and variables such as time window, would increase the complexity to compute the optimal solution. The TPP with ETTW discussed in our paper is a large scale complex combinatorial optimization problem, and the major goal of the dispatchers turns to find the best possible platform track assignment plan within limited computation time rather than find an optimal solution that takes a long computation time. Thus, we designed a rule-based metaheuristics, a kind of approximation algorithm, to find the good quality solution within reasonable amount of computation time.

Firstly, we can transfer the rule-based metaheuristic into scheduling algorithm based on dispatching rules by eliminating Step 5 in Algorithm 1. To further increase the RS feasibility, the dispatchers usually use the platform track changing strategy and intra-group swap strategy in Step 5 in Algorithm 1.

Rather than simply changing the route in the alternative inbound (outbound) route set to avoid conflicts, the dispatchers are more focused on changing platform track assignment, and the inbound or outbound route that is dependent on platform track will also be changed.

The same type of trains can be allocated to one group set and assigned to the same alternative platform track set because they have similar operating procedures and service requirement of platform facilities. Conflicts are more likely to happen between those adjacent trains in one group set due to the same alternative platform track and route set. Therefore, dispatchers usually swap platform track assignment of adjacent trains in one group set or swap train processing list orders in one group set to generate a new solution, especially for those group sets with unassigned trains. By swapping the processing list order of unassigned train A with the order of another train B in the same group set, the dispatcher can give unassigned train a higher priority to occupy limited station resources, which gives higher possibility to reduce the number of unassigned trains.

As discussed in Section 4, we also adjust the arrival and departure times of unassigned trains within ETTWs in Step 5 of Algorithm 1 to increase the possibility of acquiring a feasible train assignment plan.

\section{Algorithm 1: Rule-based metaheuristic for TPP} Input: All the basic parameters listed in Table 1. UPC and time windows.

Output: the best possible platform track assignment plans, and values of objective functions.

Step 1: Initialization

Divide train set $\mathrm{J}$ into subsets. $o_{i k}=0, o_{i k l}=0, o_{i p}=0$, $o_{i k l q}=0, \rho_{k}=0, D_{i}=1$.

Step 2: Calculation of parameters

For each train in set $\mathbf{J}$, compute claim and release time instants of the alternative platform tracks and track-circuits in all the alternative inbound or outbound routes.

Step 3: Sequencing for waiting-processing jobs

Select dispatching rules in Section 5.1 and work out the initial processing sequence list $\mathbf{J}$ ' for all waiting-processing jobs.

Step 4: Check the conflicts

For each train on list J', call Algorithm 2 to assign relative platform track, platform, inbound route, outbound route, track-circuits, and compute objective values of RSs.

Step 5: Adjustment and optimization

Step 5.1: Current and best-so-far solutions

Generate new incumbent solutions by adopting the solution improvement strategies and calling Algorithm 2 to check the conflicts. If $\Gamma \neq \varnothing$, adjust unassigned trains actual time $x_{i}^{*}$ or $y_{i}^{*}$ with a short time interval (i.e. 10 seconds) each time and adjust the claim and release time instants of related resources. Call Algorithm 2 to recheck the conflicts.

If the new valid solution is superior to the best-sofar one, then replace the latter one with the former one, and also renew processing sequencing list and values of objective function. Otherwise, the new incumbent solution is accepted in a certain probability to make the metaheuristic escapes from local optimal solution and enhance the global search ability. 
Step 5.2: Judgment of algorithm termination conditions

If algorithm termination conditions are satisfied, the algorithm ends with the best-so-far solution as the good quality solution. Otherwise, go to Step 5.1 .

\section{Algorithm 2: Conflicts check algorithm}

Input: train $i$.

Output: platform track, platform, inbound route, outbound route assigned to train $i$ and their claim and release time instants.

\section{Step 1: Select a platform track}

Select a platform track $k$ in set $Q_{j}$. If no more platform track $k$ existed in the set $Q_{i}$ can satisfy the constraint that platform track $k$ should be clear during the occupation time interval $[S(i, k), F(i, k)]$, then $J_{i} \in \Gamma$, $D_{i}=1 ; o_{i k}=0$; and reasons for not being assigned are also recorded. End Algorithm 2.

If $J_{i} \in \mathbf{J}_{\text {or }}\left(Q_{i k}^{a} \neq \varnothing\right)$, then go to Step 1.2; otherwise go to Step 1.1.

Step 1.1: Platform track and inbound route assignment

Select an inbound route $I$ in set $Q_{i k}^{a}$. To assign platform track $k$ in set $Q_{i}$ and inbound route $I$ in set $Q_{i k}^{a}$ to a given train $i$, the condition that all the track-circuits $q$ in inbound route $/$ should be clear during the occupation time interval $\left[S\left(i, l, q, o_{i k}\right), F\left(i, l, q, o_{i k}\right)\right]$ should be satisfied.

If feasible inbound route in set $Q_{i k}^{a}$ does not exist, select a new platform track in Step 1 . If there is an inbound route I in set $Q_{i k}^{a}$ satisfying the above conditions, then platform track $k$ and inbound route $/$ are assigned to given train $i$. There are the following two conditions: Step 1.1.1: If $J_{i} \in J_{t e}\left(Q_{i k}^{d} \neq \varnothing\right)$, then $\rho_{k}=\rho_{k}+1 ; D_{i}=0 ; o_{i k}=1$; $o_{i k l}=1$; and $o_{i k l q}=1\left(\forall S_{q} \in S_{\text {Set }}\right)$. Go to Step 2 .

Step 1.1.2: If $J_{i} \in \mathbf{J}_{t u} \cup \mathbf{J}_{s t} \cup \mathbf{J}_{n s}$, then go to Step 1.2. Step 1.2: Outbound route assignment

Select an outbound route e in set $Q_{i k}^{d}$. To assign outbound route e in set $Q_{i k}^{d}$, for given train $i$ all track-circuits $q$ in outbound route $e$ should be clear during the occupation time interval [S(i,e,q, $\left.\left.o_{i k e}\right), F\left(i, e, q, o_{i k e}\right)\right]$.

If feasible outbound route in set $Q_{i k}^{d}$ does not exist, select a new platform track in Step 1. If there is an outbound route $e$ in set $Q_{i k}^{d}$ satisfying the above condition, then there are the following two conditions:

Step 1.2.1: If $J_{i} \in J_{o r}\left(Q_{i k}^{a} \neq \varnothing\right)$, then outbound route $e$ is assigned to train $i ; \rho_{k}=\rho_{k}+1 ; D_{i}=0 ; o_{i k}=1 ; o_{i k e}=2$; and $o_{i k e q}=1\left(\forall S_{q} \in S_{e} t_{e}\right)$. Go to Step 2 .

Step 1.2.2: If $J_{i} \in \mathbf{J}_{t u} \mathbf{J}_{s t} \mathbf{J}_{n s}$, then outbound route $I$ is assigned to train $i ; \rho_{k}=\rho_{k}+1 ; \quad D_{i}=0 ; \quad o_{i k}=1 ; \quad o_{i k l}=1$; $o_{i k l q}=1\left(\forall S_{q} \in S_{\text {Set }}\right) ; o_{i k e}=2 ;$ and $o_{i k e q}=1\left(\forall S_{q} \in S_{e} t_{e}\right)$. Go to Step 2.

Step 2: Occupation time interval of selected platform track

Compute the incumbent total occupation time of platform track $k$, then $\sum_{z=1}^{\rho_{k}} t_{z}^{k}=\sum_{z=1}^{\rho_{k}} t_{z}^{k}+F(i, k)-S(i, k)$.

\section{Step 3: Platform assignment}

If $o_{i k}=1$, then platform $p$ which satisfies the mapping relationships $\mathbf{M}-\mathbf{P}$ is also claimed by the given train $i$, namely $o_{i p}=1$.

\section{COMPUTATIONAL TESTING AND RESULTS}

In this section, we verify the above model and algorithm through experimental study of TPP in a busy complex passenger station and give the results of testing. Based on models and algorithms proposed in this paper, we have developed a Platform-track Utilization Decision Support System (TUDSS) by Visual Basic, C\# and secondary development for AutoCAD. The system has been used in several passenger stations, i.e. Guangzhou East Railway Station.

\subsection{Data used in computational testing}

As shown in Figure 2, the Guangzhou East Railway Station has 14 platform tracks, 7 platforms, 4 lines in Shenzhen direction and 2 lines in Guangzhou direction. In the interlocking chart of the station, there are 317 inbound and outbound routes and 105 track-circuits. The station has 264 arrival and departure trains in the timetable from $18: 00 \mathrm{pm}$ to $18: 00 \mathrm{pm}$ on the very next day, including 86 THPTs, 74 pairs of ORPTs and TEPTs and 15 pairs of ATUPTs and DTUPTs according to carriages turnover plan. The schematic layout for Guangzhou East Railway Station includes directions, platforms and so on.

\subsection{Results and analysis}

\subsubsection{The impact of dispatching rules on solutions}

For TPP in Guangzhou East Railway Station, the objective values for RSs are illustrated in Table 5.

In Table 5, it can be concluded that RSs can be acquired within 3,000 ms. The average sub-objective values of different dispatching rules are illustrated in Figure 3. For the weighted number of delayed trains, rule 7 provides a much better solution than those of any other rules, and also we can see that the RS based on rule 7 is around $30 \%$ better than the one based on rule 8 (FIFO-EDD) that is commonly used in existing papers such as Carey (2003) [9]. For the platform track preference, rules 6 and 12 provide better solutions than those of any other rules. The RS based on rule 5 provide worst solution for the weighted number of delayed trains, but it provides the best solution for the balanced use of resources. Thus, we can found that disparities between the RSs based on different rules are quite obvious in a busy complex passenger station and that the same dispatching rule may provide different results for different sub-objectives. If we set 100 iterations (around 4 minutes) as termination condition 


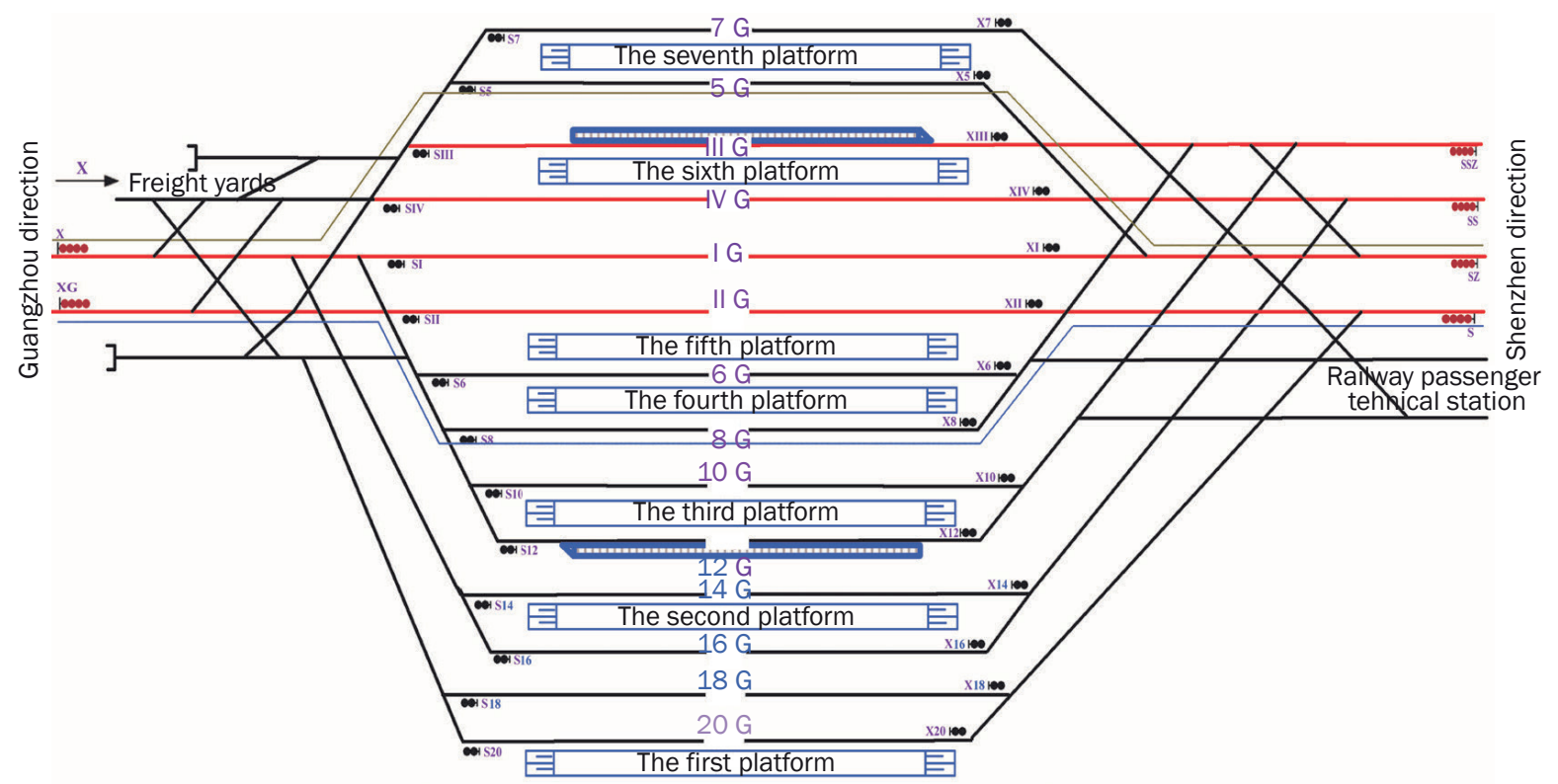

Figure 2 - Schematic layout for Guangzhou East Railway Station

Table 5 - RSs for MCS

\begin{tabular}{||c|c|c|c|c|c|c||}
\hline \multirow{2}{*}{ No. } & Dispatching rules & \multirow{2}{*}{$\begin{array}{c}\text { Average com- } \\
\text { puting time } \\
{[\mathrm{ms}]}\end{array}$} & $\begin{array}{c}\text { Weighted num- } \\
\text { ber of delayed } \\
\text { trains }\end{array}$ & $\begin{array}{c}\text { Platform track } \\
\text { preference }\end{array}$ & $\begin{array}{c}\text { Balanced use } \\
\text { of resources }\end{array}$ & $\begin{array}{c}\text { Average objec- } \\
\text { tive value [min] }\end{array}$ \\
\hline \hline 1 & EDD & 2,137 & 346.6 & 276.5 & $1,108.2$ & 370.7 \\
\hline 2 & FIFO & 2,355 & 395.7 & 285.1 & $1,019.5$ & 409.8 \\
\hline 3 & SPT & 2,123 & 264.6 & 401.6 & $1,251.0$ & 341.3 \\
\hline 4 & WSPT & 2,276 & 323.2 & 371.7 & $1,247.9$ & 379.1 \\
\hline 5 & WDSPT & 1,984 & 646.4 & 346.4 & 912.1 & 599.6 \\
\hline 6 & BW & 2,785 & 419 & 254.4 & $1,370.7$ & 418.6 \\
\hline 7 & SL & 2,459 & 200 & 368.2 & $1,182.3$ & 282.7 \\
\hline 8 & FIFO-EDD & 2,068 & 400 & 274.3 & $1,023.7$ & 406.2 \\
\hline 9 & EDD-FIFO & 2,065 & 359 & 287.6 & $1,118.1$ & 382.7 \\
\hline 10 & WSPT-EDD & 2,165 & 286.8 & 329.5 & $1,248.5$ & 343.4 \\
\hline 11 & WSPT-FIFO-EDD & 2,142 & 297.2 & 338.1 & $1,247.7$ & 352.9 \\
\hline 12 & SL-FIFO-EDD & 2,297 & 517.6 & 256.5 & $1,007.4$ & 489.8 \\
\hline 13 & BW-SL-FIFO-EDD & 2,035 & 423.2 & 392.5 & $1,054.3$ & 448.6 \\
\hline 14 & SL-BW-FIFO-EDD & 2,354 & 580.2 & 299.3 & 964.1 & 543.2 \\
\hline 15 & -FIFO-EDD & 2,788 & 528 & 350.93 & 989.5 & 515.6 \\
\hline
\end{tabular}

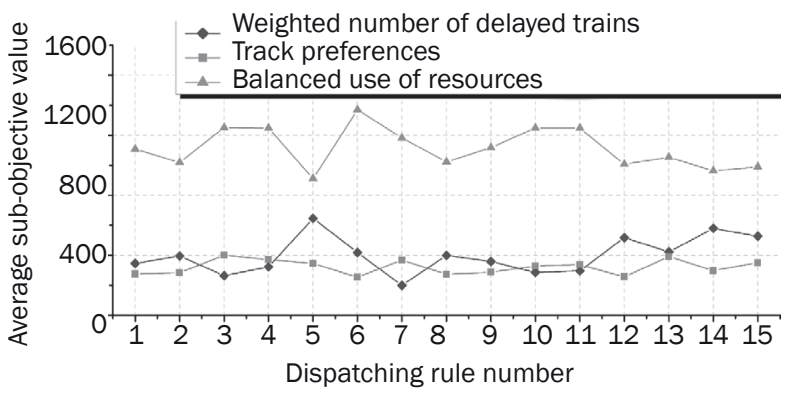

Figure 3 - Sub-objective values of RSs and the subobjective values of RSs in MCS without ETTW in Step 5.2 of Algorithm 1, the good quality solution of metaheuristics is shown in Table 6.

The good quality solutions of MCS is around 14\% better than the RSs of MCS, which proves that the combination of two solution improvement strategy is effective to optimize the RS in limited computation time. Within short computation time, the good quality solution based on rule 7 with combined solution improvement strategy is around $25 \%$ better than the good quality solution of rule 8 with platform changing strategy which is commonly used in existing papers. Therefore, the impact of RS on the good quality 
Zeng Q, Zhang Y, Lei D. An Experimental Analysis on Dispatching Rules for the Train Platforming Problem in Busy Complex Passenger...

Table 6 - Comparison between RSs of MCS and the good quality solution solutions of MCS (100 iterations)

\begin{tabular}{||c|c|c|c||}
\hline \hline Rule No. & RS & Good quality solution of combined strategies & $\begin{array}{c}\text { Good quality solution of platform track } \\
\text { changing strategy }\end{array}$ \\
\hline \hline 1 & 370.7 & 336.29 & 347.01 \\
\hline 2 & 409.8 & 371.53 & 359.53 \\
\hline 3 & 341.3 & 307.15 & 317.61 \\
\hline 4 & 379.1 & 308.82 & 333.3 \\
\hline 5 & 599.6 & 480.11 & 470.64 \\
\hline 6 & 418.6 & 355.44 & 309.73 \\
\hline 7 & 350.2 & 276.55 & 306.41 \\
\hline 8 & 406.2 & 363.69 & 368.31 \\
\hline 9 & 382.7 & 342.29 & 324.62 \\
\hline 10 & 343.4 & 316.59 & 300.78 \\
\hline 11 & 352.9 & 320.73 & 320.71 \\
\hline 12 & 489.8 & 370.92 & 360.68 \\
\hline 13 & 448.6 & 425.75 & 420.47 \\
\hline 14 & 543.2 & 387.74 & 359.33 \\
\hline 15 & 515.6 & 414.76 & 381.42 \\
\hline
\end{tabular}

solution under the same rule is great in busy complex passenger stations within limited computation time and combined solution improvement strategy is of significant importance.

In the following analysis, we will analyse the weighted number of delayed trains as the first and most important sub-objective. Figure 4 compares good quality solutions of MCS and TWS under different dispatching rules if we set 35 min computing time as termination condition in Step 5.2 of Algorithm 1. The illustration compares the good quality solutions of MCS and good quality solutions of TWS under different dispatching rules.

For different dispatching rules, the good quality solutions for TWS are generally better than those for MCS, and the former are around 15\% better than the latter in terms of their good quality solutions on the

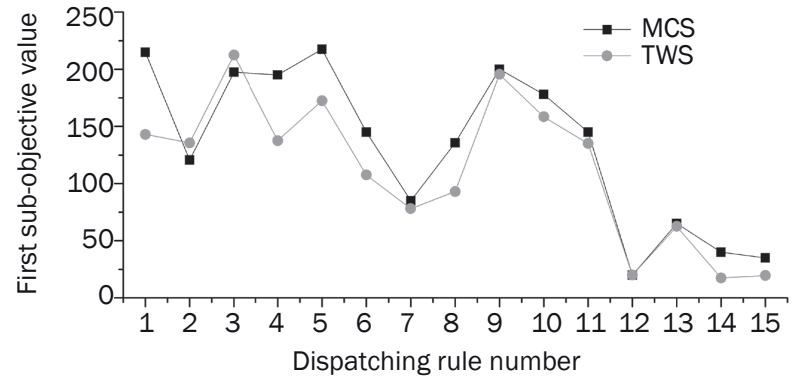

Figure 4 - The good quality solutions comparison between MCS and TWS

average level, respectively. For instance, the solution convergent speed for TWS is much slower under dispatching rule 14 (cf. Figure 5). In Figure 5, the horizontal axis represents the computing times while the vertical axis represents variation of the first sub-objective

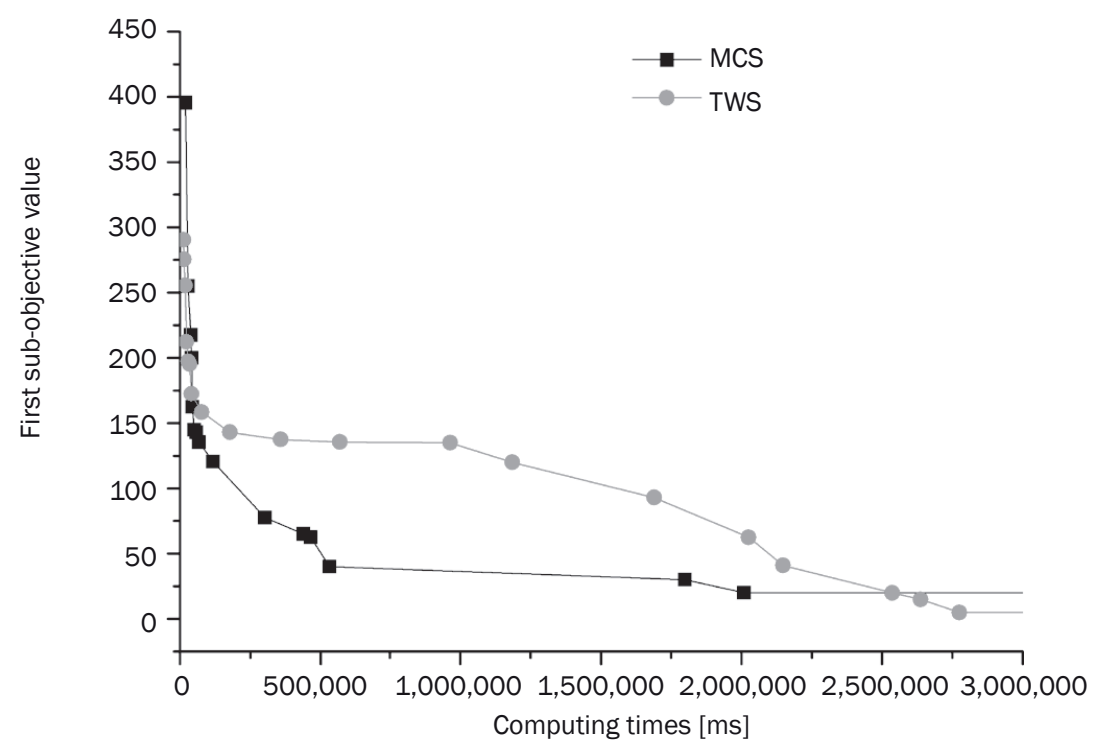

Figure 5 - Solution convergent speed comparison between MCS and TWS 
value of best-so-far solution with computing times. When computing times are longer than 3,000,000 ms, the good quality solution of TWS is better than the good quality solution of MCS as shown in Figure 5. Therefore, we had better choose TWS with ETTW when we make long-term platform track assignment plans such as daily plans ( 24 hour) and shift plans (12 hour). When computing times are less than 3,000,000 ms, the good quality solution of MCS is better than the good quality solution of TWS as shown in Figure 5 . The acceptable time for making a stage plan (4-hour or 3-hour platform track assignment plan) is about 20 minutes (around 1,200,000 ms in Figure 5). Suitable dispatching rules that can achieve a good RS of MCS (TWS) definitely will help us get a better good quality solution of MCS (TWS) within limited computation time. Therefore, we had better choose MCS for TPP without considering ETTW and choose suitable dispatching rules to access a relatively good solution within limited computation time when we make short-term platform track assignment plans. The illustration compares the solution convergent speed of MCS and TWS under dispatching rule 14 .

\subsubsection{Expandability analysis of the proposed approach}

The passenger stations in China can be categorized into high-speed railway stations and existing passenger stations. High-speed railway stations are featured by single train type, high platform and shorter dwell time (i.e. 2 min). However, the existing passenger stations have many various train types, different types of platforms and longer dwell times.

Furthermore, we have tested the model and algorithm in a station in High-speed Railway network. Tielin West station is a high-speed railway station with two directions, six tracks and four platforms. In timetable of the station, there are 82 arrival and departure trains, including 28 THPTs and 26 pairs of ORPTs and TEPTs. For TPP in Tielin West Railway Station, we can find that the average objective values of RSs (11.45 min) are the same for Tielin West Railway Station which is sparse in arrivals and departures, simple in station layout and single in train types. The only difference between solutions based on different dispatching rules is the average computing time, i.e. the average computing time for dispatching rules 1, 3 and 15 are 566, 965, 1,332 mm, respectively. For TPP in Guangzhou East Railway Station, the average objective values of RSs are illustrated in Table 4 . In busy passenger stations with more intense occupation of platform tracks, the number of unassigned trains increases with density of arrivals and departures in timetable because the platform track and route resources are more limited. Thus, the order in which trains is considered when assigning platform tracks may cause higher degree of delay, and differences between the average objective values of RSs are much more obvious (as shown in Table 4) in passenger stations with more intense occupation of platform tracks.

Also, some of the stations in other countries, i.e. UK, have platform tracks that can accommodate more than one train at the same time; some trains can be cancelled occasionally in advance; and most passengers can take many trains at certain station by "peaktime tickets" or "off peak-time tickets". In China, trains cannot be cancelled except for the nature disasters and other uncontrollable reasons, because passengers must take a given train at certain time interval and certain station according to their tickets. Although there are more strict limitations in China than in other countries, the proposed dispatching rules can be also applied into other different passenger stations both in China and other countries.

\section{CONCLUSIONS}

In this paper, we identified TPP in busy complex passenger stations as special cases of job shop scheduling problem and presented track-circuits based formulations for TPP. Firstly, we represent different operations of different train types conducted in complex passenger stations and propose a microscopic simulation of train movements in interlocking area, which is necessary for track-circuited formulation of TPP specific to complex stations. Secondly, specific to complex stations, we built MCS with an objective that combined sub-objective of platform track preferences with sub-objective of balanced use of platform tracks. Thirdly, we built TWS to formulate the TPP in operational level and explicitly represented the UPCs of different train types. Fourthly, we design dispatching rules by incorporating classic scheduling theory with experience of dispatchers and compared the RSs based on those rules. Fifth, we also introduced combined solution improvement strategies analogous to manual method used in practice by dispatchers in the rule-based metaheuristic to get good quality solution within limited computation time.

Notably, we can also explore the impact of dispatching rules on the sub-objective of robustness. Further, we will try to integrate the timetable of placing-in and taking-out operations with the timetable of arrival and departure operations in the future research.

\section{ACKNOWLEDGEMENT}

The work described in this paper was support by grants from the National Natural Science Foundation of China (Nos. 71501190, 71371193). This work was also supported in part by the scholarship from China Scholarship Council (No. 201606375002). The authors declare that there is no conflict of interest regarding the publication of this paper. 
曾琼芳, 博士研究生, 中南大学交通院运输工程学 院, 湖南 长沙, 410075

张英贵 $*$, 博士, 讲师, 中南大学交通院运输工程学 院, 湖南 长沙, 410075

雷定猷 $*$, 博士, 教授, 中南大学交通院运输工程学 院, 湖南 长沙, 410075

\section{繁忙复杂客运站列车停靠站台问题的调度规则试验 分析}

摘要

采用混合整数线性规划和机器排序理论, 论文构建 了一个列车停靠站台问题 (TPP) 的排序模型。首 先，通过阐述繁忙复杂铁路客运站不同类型列车的 作业过程, 给出了不同类型列车时间调整成本的计 算方法。然后, 分别设计了不考虑早晚点时间窗

(ETTW) 约束的多目标排序模型 (MCS) 和带早晚点 时间窗 (ETTW) 约束的窗时排序模型（TWS）。最 后, 统筹考虑车站调度员的人工经验和现代排序理 论，设计诸多调度规则，并提出基于规则的启发式 算法求解上述模型。计算结果表明: 使用基于车站 调度员现场处置经验的解改进优化策略, 论文所提 出的方法能够在可接受的时间内有效解决铁路现场 大规模的列车停靠站台问题

\section{关键词}

繁忙复杂客运站，列车停靠站台问题，机器排序理 论, 调度规则

\section{REFERENCES}

[1] Sels P, Vansteenwegen P, Dewilde T, Cattrysse D, Waquet $B$, Joubert $A$. The train platforming problem: The infrastructure management company perspective. Transportation Research Part B Methodological. 2014;61(1):55-72.

[2] Dewilde T, Sels P, Cattrysse D, Vansteenwegen P. Improving the robustness in railway station areas. European Journal of Operational Research. 2014;235(1):276-286.

[3] Zhou WL, Teng HL. Simultaneous passenger train routing and timetabling using an efficient train-based Lagrangian relaxation decomposition. Transportation Research Part B. 2016;94:409-439.

[4] Cacchiani V, Galli L, Toth P. A tutorial on non-periodic train timetabling and platforming problems. Euro Journal on Transportation \& Logistics. 2014;4(3):1-36.

[5] Mascis A, Pacciarelli D. Job-shop scheduling with blocking and no-wait constrains. European Journal of Operational Research. 2002;143(3):498-517.

[6] D'Ariano A, Pacciarelli D, Pranzo M. A branch and bound algorithm for scheduling trains in a railway network. European Journal of Operational Research. 2007;183(2):643-657.

[7] Samà M, Meloni C, D'Ariano A, Corman F. A multi-criteria decision support methodology for real-time train scheduling. Journal of Rail Transport Planning \& Management. 2015;5(3):146-162.

[8] Corman F, Goverde R, D'Ariano A. Rescheduling dense traffic over busy complex station interlocking areas. In: Ahuja R, Möhring R, Zaroliagis C (Eds.). Robust and Online Large-Scale Optimization: Models and Techniques for Transportation Systems. Berlin, Germany: Springer-Verlag Berlin Heidelberg, 2009; p. 369-386.

[9] Carey M, Craville S. Scheduling and platforming trains at busy complex stations. Transportation Research Part A: Policy \& Practice. 2003;37(3):195-224.

[10] Carey M, Crawford I. Scheduling trains on a network of busy complex stations. Transportation Research Part B: Methodological. 2007;41(2):159-178.

[11] Fang W, Yang S, Yao X. A survey on problem models and solution approaches to rescheduling in railway networks. IEEE Transactions on Intelligent Transportation Systems. 2015;16(6):2997-3016.

[12] Lusby RM, Larsen J, Ehrgott M, Ryan DM. A set packing inspired method for real-time junction train routing. Computers \& Operations Research. 2013;40(3):713724.

[13] Pellegrini P, Marlière G, Rodriguez J. Optimal train routing and scheduling for managing traffic perturbations in busy complex junctions. Transportation Research Part B: Methodological. 2014;59(1):58-80.

[14] Alagöz O, Azizoğlu M. Rescheduling of identical parallel machines under machine eligibility constrains. European Journal of Operational Research. 2003;149(3):523-532.

[15] Caprara A, Galli L, Toth P. Solution of the train platforming problem. Transportation Science. 2011;45(2):246257.

[16] Caimi G, Fuchsberger M, Laumanns M, Lüthi, M. A model predictive control approach for descrete-time rescheduling in busy complex central railway station approach. Computers \& Operations Research. 2012;39(11):145-155.

[17] Dewilde T, Sels P, Cattrysse D, Vansteenwegen P. Robust railway station planning: An interaction between routing, timetabling and platforming. Journal of Rail Transport Planning \& Management. 2013;3(3):68-77.

[18] Pinedo M. Scheduling theory, algorithms and systems. New York, NY: Springer Science+Business Media, LLC; 2008.

[19] Kroon LG, Romeijn HE, Zwaneveld PJ. Routing trains through railway stations: busy complexity issues. European Journal of Operational Research. 1997;98(3):485-498. 